\title{
Study on temporal and spatial variation regularity of soil moisture content based on interpolation algorithm
}

\author{
Liying Cao, Tengda Qie, Guifen Chen \\ School of Information Technology, Jilin Agricultural University , Changchun 130118 \\ caoliying99@163.com
}

Keywords: soil moisture; time; temperature and humidity; Jilin Province

\begin{abstract}
ArcGIS environment through IDW interpolation method analysis of the spatial variation of water content in soil, and soil moisture content of the internal and seasonal variations, discusses the precipitation and temperature on the effect of soil moisture content changes. The results showed that the Nongan County, Jilin Province Experimental Zone in the growth period, with little change in soil temperature, and soil moisture change obvious; soil moisture in at the end of June was the lowest in July, around the four content. Soil temperature in June around the 10th lowest in July 10 about the highest temperature; precipitation is affecting the province's soil containing the dominant factors of the temporal and spatial variation of water and temperature had little effect. The precipitation depends on the soil moisture level.
\end{abstract}

\section{Introduction}

From the global development of the status quo, the United States, Canada, the United Kingdom in the most mature application of fertilization, the basic entry into commercial use. Japan mainly focuses on the research of the sensor and the automatic control of the agricultural machinery. Precision agriculture is also widely used in forestry production, mainly related to the fertilization, precision seeding, disease and insect pest control, harvesting and water management related fields. At present, except for a few countries, precision agriculture in the world has not yet large-scale deployment, main reason is its key technology development has not yet in practical value produce a breakthrough, information acquisition technology, and the cost is expensive, but the international of precision agriculture technology development potential and application prospects have broad consensus, its important content as a developing agricultural high and new technology application, said the agricultural sustainable development is an important way.

\section{Research areas and research methods}

precision operating system development and application of "demonstration base of Nong'an County Helong Zhen, Nong'an county at Songliao plain, is one of the important commodity grain base, Jilin Province, located 60 kilometers northwest of Changchun City, east longitude 124 degrees 32 '- 125 degrees 45'. - 44 degrees north latitude 43 degrees 54 '56'; average annual precipitation $507.7 \mathrm{~mm}$, mainly soil fertility grade chernozem soil, black soil and alluvial soil, aeolian sandy soil, saline soil, alkaline earth, swamp soil, peat soil and paddy soil. Black Nong'an county the most fertile soil of, concentrated in the county of eastern and southern Bao Jia, Helong, pot roast, patron and three Gang Township (town) of the Loess in product platform, in our province black soil zone edge, climate close to semi-arid region.

According to maize precision production objective laws, using Kriging interpolation method of topography, soil moisture and discrete sampling data interpolation into regular volume data, spatial interpolation map generation, for 3D reconstruction; image show the regular grid data, need to different depth of soil moisture and soil temperature data were interpolated, including light and shade effect of interpolation and application to meet the display area of each pixel for rendering the spatial variability.

Analysis of three-dimensional space interpolation map 
Apply of three-dimensional space processing technology, on topography and different depth of soil moisture, soil temperature spatial variation diagram for a variety of combinations. Analysis of the relationship among the various factors of soil moisture, topography, soil temperature, to solve the complex of soil moisture monitoring and spatial analysis problems.

\section{Temporal and spatial variation regularity of soil moisture content based on interpolation algorithm research}

This paper uses a Mann-Kendall (MK) rank correlation test method, analysis the trend of soil water variation. For the lack of measured data, using SPSS software based on self correlation interpolation site. The correlation between soil moisture and precipitation and temperature is calculated by SPSS software. Through the GIS platform, using inverse distance weighted interpolation method (Inverse Distance Weighting, IDW) in Jilin Province, drawing soil water, precipitation and temperature of the monthly average spatial variation (Figure 1). IDW interpolation method does not consider the differences in underlying surface types, so the interpolation result has some limitations, such as slope, irrigation etc. the interpolation results and the actual situation may be quite different. To analyze spatial distribution rules of soil water in Jilin Province, according to the soil moisture for command in the size of water using GIS software of soil water were classified, divided into three grades (the system to generate terrain model is mainly concerned with the building up of the model of ground and underground. First of all, based on the boundary demarcation and Nong'an grades were arc map drawing fertility.

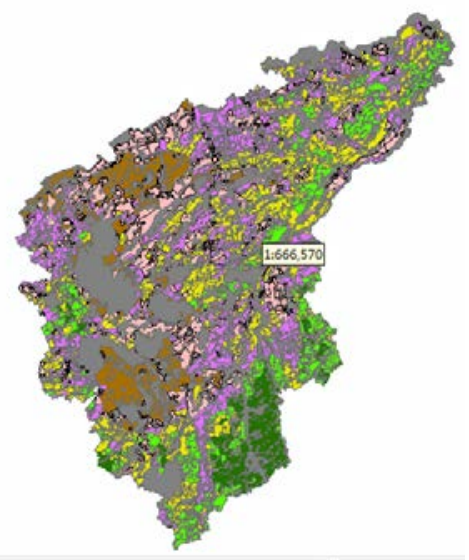

Fig. 1 distribution map of soil fertility level in Nong'an County

Temperature distribution of soil moisture in maize growing period

According to the dynamic monitoring of $0-20 \mathrm{~cm}, 20-40 \mathrm{~cm}, 40-60 \mathrm{~cm}, 60-80 \mathrm{~cm}$ four levels of soil moisture, soil moisture, soil temperature data, soil moisture, temperature change trend chart as shown in figure 2- figure 5.

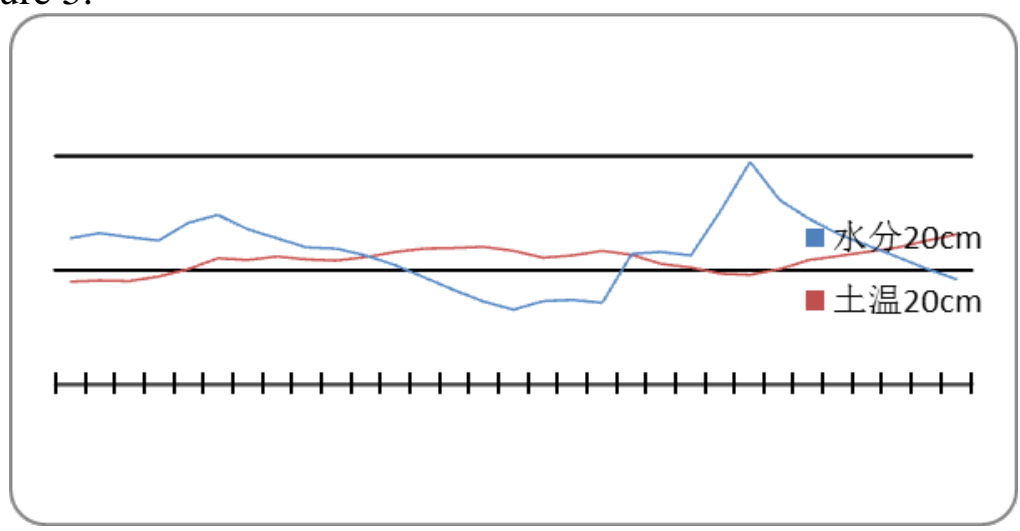

Fig. 2 change trend of soil temperature and moisture in crop growth period $0-20 \mathrm{~cm}$ 


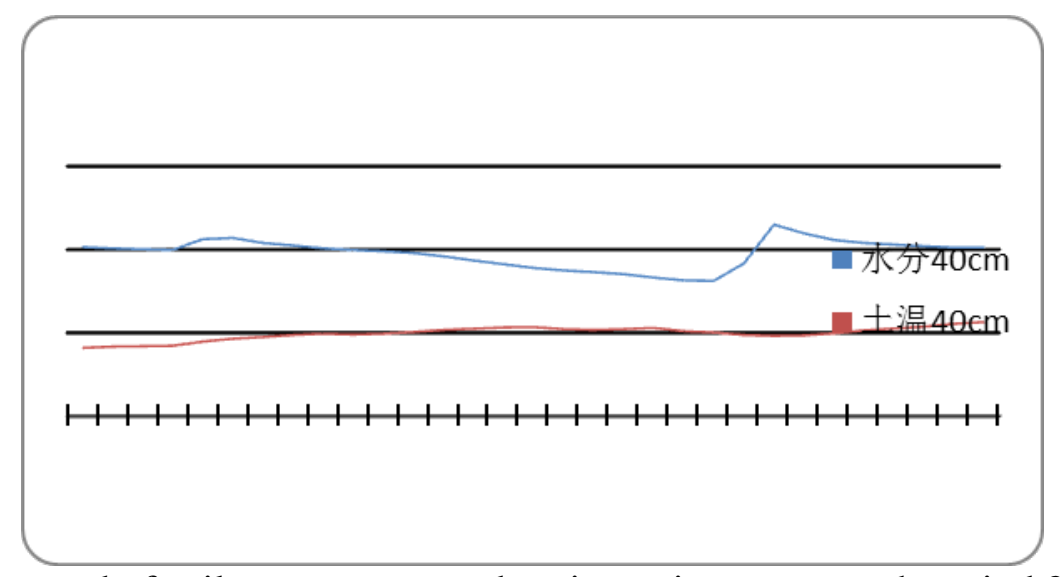

Fig. 3 change trend of soil temperature and moisture in crop growth period $20-40 \mathrm{~cm}$

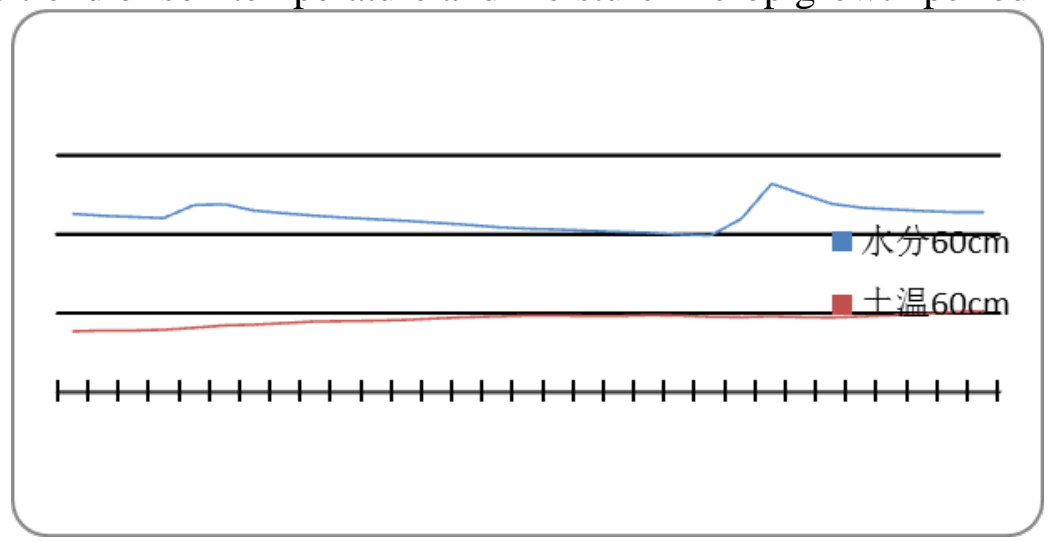

Fig. 4 change trend of soil temperature and moisture in crop growth period $40-60 \mathrm{~cm}$

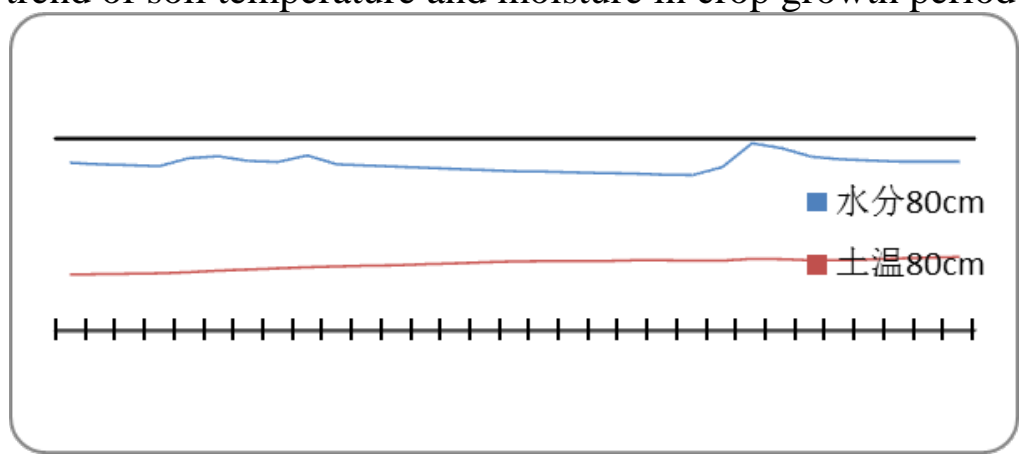

Fig. 5 change trend of soil temperature and moisture in crop growth period $60-80 \mathrm{~cm}$

From Fig. 2 - 5 can be seen in the growth period, with little change in soil temperature, and soil moisture change obvious; soil moisture in at the end of June was the lowest in July, around the four content. Soil temperature in June around the 10th lowest in July around the 10th highest temperature;

\section{Discussion}

Beginning in March, the water content of the soil decreased slowly, reaching the minimum value after reaching the minimum value in June. After 9 and October, the soil water content increased slowly. Variability in soil moisture can be divided into four stages: Spring slowly consuming stage, summer rapid increase moisture period, increase in autumn transition period and winter steady moisture period. Precipitation is the dominant factor that affects the spatial and temporal variation of soil moisture in Jilin Province, and the second is the temperature. Apart from the central and western regions, the remaining 3 areas of precipitation and soil water in a large annual correlation. Air temperature and soil water in the annual correlation is limited by the amount of precipitation. Generally, the soil moisture content change with the amount of precipitation, the temperature internal variability of water on the soil moisture has certain influence, annual variation has little effect, space impact is not great. 


\section{Acknowledgments}

This work was funded by The key project of the national Spark Program in 2015(2015GA660004) , Science and technology development project of Jilin Province(20160412034XH).

\section{References}

[1] Zhang Haoran. GIS technology in the mobile field [J]. mapping and spatial geographic information, 2014,37 (4): 137-138141.

[2] Alkevli Tolga, Ercanoglu Murat. Assessment of ASTER satellite images in landslide inventory mapping: Yenice-Gok5ebey(Western Black Sea Region, Turkey)[J]. Bulletin of Engineering Geology and the Environment, 2011, 70(4):607-617.

[3]Janmes Swanson,The Three Dimensional Visualization\&Analysis of Geography Data[EB/OL],httP://www.maPs.unomaha.eduP/etersonjGis/Final-Projeets/1996,2002-11-10.

[4] Li Kuixing, the overall design and visualization of 3D geological spatial information system [D], Ph. D. Thesis, Peking University, Beijing: Peking University, 2000

[5] C Yu Zhiwei, Xiaoping, Xu Youzhi, on the construction of three-dimensional GIS thinking [J], geology and exploration, 2001,37 (4): 63-67.

[6] Huang Haizan, stereo vision and 3D reconstruction method research [D], Shanghai Jiao Tong University, Bo ten degree thesis, Shanghai: Shanghai Jiao Tong University, 2001

[7] Yao Xin, Wang Chongchang. Establishment and visualization of 3D model of mineral deposit based on ArcScene: [J]. engineering geology computer application, 2006 (1): 125-126. 